\title{
Experimental research on degradation performance of magnesium alloy affected by mechanical environment,
}

\author{
Dong Limin ${ }^{1}$, Song Xinxin ${ }^{1}$, Ye Jinduo ${ }^{1}$, Zhang Chunqiu ${ }^{1}$, Liu Nian $^{2}$, Chen Wei $^{2}$ \\ ${ }^{1}$ Tianjin Key Laboratory of the Design and Intelligent Control of the Advanced Mechatronical System, Tianjin University of Technology, School \\ of Mechanical Engineering, Tianjin, 300384, China \\ ${ }^{2}$ Tianjin Just Huajian Medical Instrument Co. , Ltd, Tianjin 300384, China
}

\begin{abstract}
Objective To research the mechanical properties of magnesium alloy degradation affected by mechanical environment. Methods: After being divided into 4 groups randomly such as No.1, 2, 3and 4,12 AZ31 magnesium alloy screws need suspending in the vessels containing $35 \mathrm{ml}$ simulated body fluid . No.1 was control group. Group 1 was under no load condition, experimental group 2, 3, and 4 were discontinuously applied fixed axial compression load of $30 \mathrm{~N}, 60 \mathrm{~N}, 90 \mathrm{~N}$ respectively on time. The weight loss of magnesium alloy screws and the amount of gas generated were measured regularly. Results: From the cumulative amount of hydrogen production, group 1 and group 4 were relatively large, whereas group 2 and group 3 were relatively small. From the weight loss of magnesium alloy screws, group $3>$ group $4>$ group $2>$ group 1 . Conclusions: The general trend is the greater the load, the faster the degradation.
\end{abstract}

Keywords:Biodegradable magnesium alloy; Mechanical environment; Experimental research

\section{Introduction}

With the development of material science and biomedicine, it is hoped that the biological material embedded in the body plays just a temporary replaced role, and with the regeneration of tissue or organs, it was gradually degraded and absorbed by the body, in order to minimize the long-term effects of materials on the body. Because biodegradable material is easy to decompose in organism, its product can metabolize and finally expel from body, which is non-toxic side effects to the body, so it attracts more and more attention [1]. Magnesium alloy, a kind of implant materials with great potential, has obvious advantages in the treatment of fracture and bone defect. First of all, magnesium alloy has good biological compatibility and biodegradability, and rigidity and intensity are higher than traditional implantable metal materials. The elastic modulus (41-45Gpa) and compressive yield strength of magnesium alloy are closer to those of natural bone (3-20Gpa). Stress conduction character is matched with bone tissue, which can avoid the "stress shielding effect" effectively [2]. Moreover, degrading appropriate amount of magnesium ions can promote osteoblast proliferating and differentiating with biological activity[3].Secondly, patients needn't second operation after recovery, which not only reduces cost, but also avoids another injury. But comparing with cobalt-chrome alloy and other metal materials,

${ }^{a}$ Corresponding author:Zhang Chunqiu,494323993@qq.com,tel:15822274830 
mechanical properties of magnesium alloy is slightly low, but corrosion rate is too high. Insufficient carrying capacity and high degradation rate will cause the failure of implant materials, and affect magnesium alloy biological materials applied in the field of clinical medicine[4].With the development of metallurgical technology and material science, the new developed magnesium alloy extends the degradation time, and still keep good biocompatibility and mechanical properties, which attracts all walks of life both domestic and overseas, and becomes one of research emphases and hotspots in the field of biomedical materials[5]. This experiment focuses on researching degradation performance of AZ31 magnesium alloy affected by mechanical environment, and provide theoretical support on clinical application of magnesium alloy bone material.

After the biological degradation, the entire strength of magnesium alloy bone internal fixation material will decline. But it is essential to ensure the strength for bone healing after the decline. Therefore, it is of great importance to study what the effects of magnesium alloy degradation factors on the degradation of magnesium alloys are. Mechanical environment impacts on magnesium alloy degradation [6.7]. In the treatment process of fracture and bone defect, bone fixation material is in a complex mechanical environment, so it is more important to study the degradation of magnesium alloy in a complex mechanical environment. Magnesium alloy electrochemical reaction produce hydrogen and $\mathrm{Mg} 2+$ in the process of degradation $[8,-10]$, it can reflect the degree of degradation of magnesium alloys by measuring hydrogen volume and magnesium alloy weight loss. This study selected magnesium alloy screws manufactured of AZ31 magnesium alloy with good comprehensive performance, by measuring produce hydrogen volume and weight loss of magnesium alloy screws in the same volume of the simulated body fluid in different mechanical environments, magnesium alloy materials degradation law in different mechanical environments was analyzed qualitatively, and provide theoretical support for the clinical application of magnesium alloy bone.

\section{The selection of experimental material and experimental method}

\subsection{Experimental material}

Select 12 AZ31 magnesium alloy material screws with $3.5 \mathrm{~mm}$ diameter, 4 syringes $(60 \mathrm{ml}$, used as a unified specification container), a number of simulated body fluid nutrient solution, color string.

\subsection{Experimental method}

(1)Control group as shown in Figuer.1: in no load state,No.1 syringe was injected with $35 \mathrm{ml}$ simulated body fluid ,immersed in which were 3 screws hanged by color string.

(2) Prepare for experimental group 2,3, and 4: syringes were injected with $35 \mathrm{ml}$ simulated body fluid respectively, 9 screws (numbered 4, 5, 6, 7, 8, 9, 10, 11, and 12 randomly) were placed in No.2, No.3, and no. 4 syringe with simulated body fluid averagely hanged by the color string. No.2 syringe was loaded the axial compression $30 \mathrm{~N}$ by the electronic universal testing machine (pressure $0.04 \mathrm{MPa}$ ) as Figuer.1, No.3 syringe $60 \mathrm{~N}$ (pressure $0.08 \mathrm{MPa}$ ), and No.4 syringe $90 \mathrm{~N}$ (pressure $0.12 \mathrm{MPa}$ ) before each of them being loaded for 1 hour, 2 times a day as the experiment group.

Put four groups of specimens at $37{ }^{\circ} \mathrm{C}$ constant temperature box in addition to the loading time. According to the normal human activity rules, gas generated was measured regularly, and weighing regularly using high precision balance (accuracy: 0.0001 g) to confirm the weight of degradation. After the experiment, the specimens were rinsed with clean water, and the morphology of magnesium alloy screws after degradation was observed by scanning electron microscope (magnification ratio were 25 times, 100 times and 500 times). The hydrogen production quantity, weight loss and other indicators were analyzed quantitatively, and the laws of magnesium alloy degradation affected by mechanical environment were found out. 

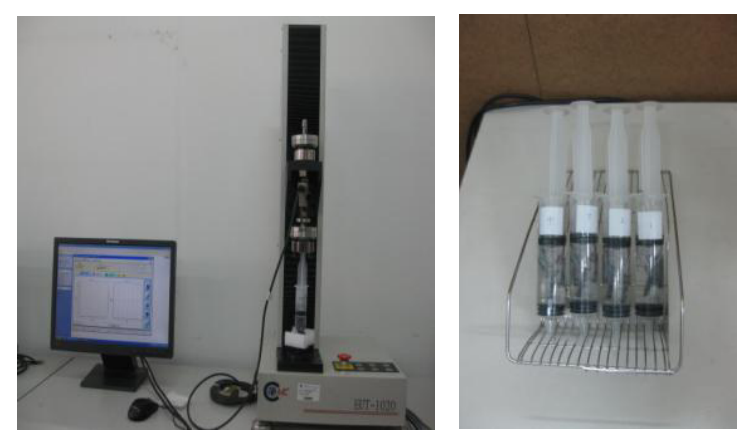

Figure.1 Electronic universal testing machine and figure of four groups Specimens

\section{Experimental results}

The experiment results are shown in figure 2 and figure 3. By comparing the experimental group 2, 3, 4 and control group 1, it is found that from the cumulative hydrogen production, the control group 1 and experimental group 4 hydrogen production amount were relatively large, the experimental group 2 and experimental group 3 were relatively small; from magnesium alloy screws weight loss are shown in figure 4 and figure5.experimental group $3>$ experimental group $4>$ experimental group $2>$ control group 1 , The degradation rate of magnesium alloy with the increase of the load is nonlinear. The degradation rate of magnesium alloy was different with different axial load. The trend was the larger the load, the faster

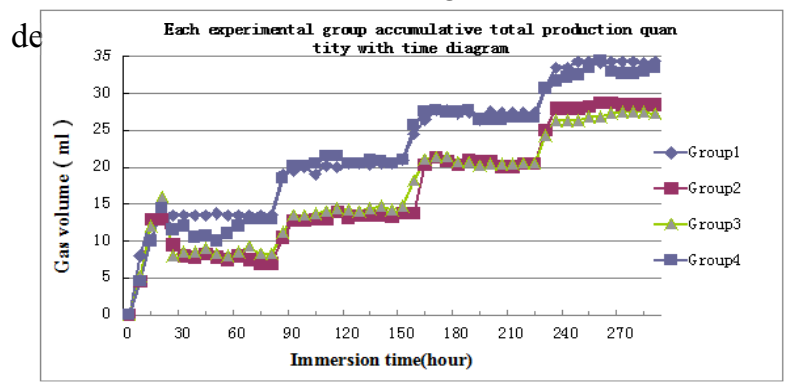

Figure.2 Each experimental group accumulative total production quantity with time diagram

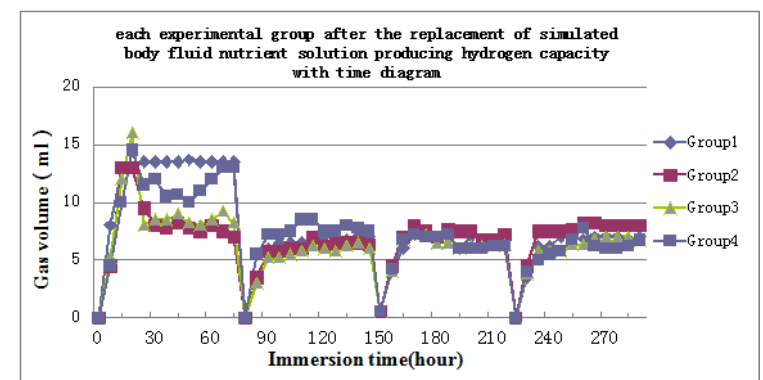

Figure.3 Each experimental group after the replacement of simulated body fluid nutrient solution Producing hydrogen capacity with time diagram

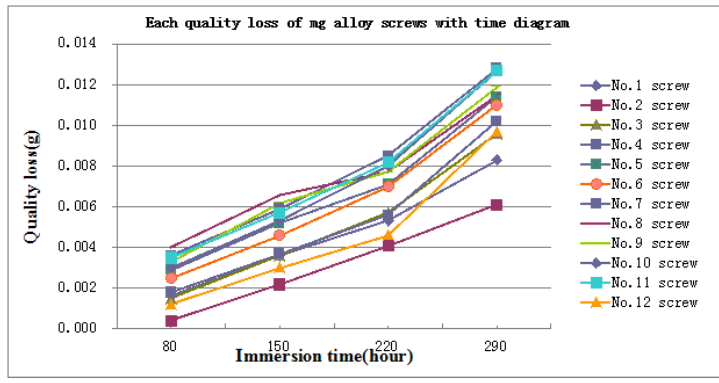

Figure.4 Each quality loss of mg alloy screws quality loss with time diagram

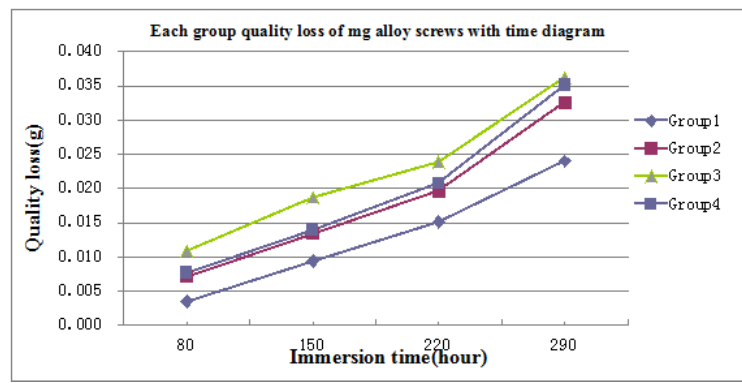

Figure.5 Each group quality loss of mg alloy screws with time diagram

Magnesium alloy degradation in vivo and in vitro is also called corrosion. The most common corrosion of magnesium alloys in simulated body fluid is galvanic corrosion, pitting, corrosion fatigue, and erosion corrosion [9]. Observed by the scanning electron microscopy after the experiment was Magnesium alloy surface morphology which was enlarged 25 times as shown respectively in a,b,c,d ,Figuer.6,enlarged 100 times as shown in e,f,g,h, Figuer.7,experimental group 2, 3,4 and the screws without degradation, what was found is that corrosion of the experimental group was more obvious than the control group. The corrosion of control group was relatively homogeneous.

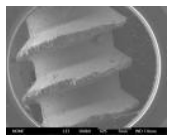

(a) Group1

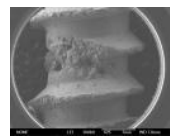

(b) Group2

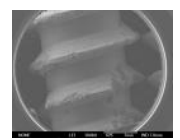

(c) Group3

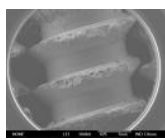

(d) Group4
Figure.6 Magnesium alloy screws enlarged 25 times by the scanning electron microscopy

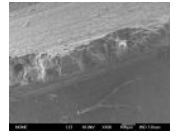

(e) Group 1

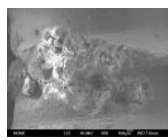

(f) Group2

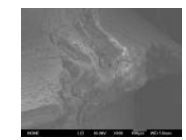

(g) Group3

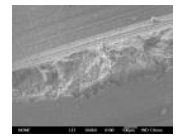

(h) Group4
Figure.7 Magnesium alloy screws enlarged 100 times by the scanning electron microscopy

Through this experiment was mainly research magnesium alloy degradation affected by mechanical environment qualitatively, so what could be drawn is that 
the mechanical environment has influence on degradation rate of magnesium alloy, magnesium alloy degradation rate is associated with the pressure of environment.

\section{Discussions}

Fractures repaired by magnesium alloy can reduce stress shelter effect in the late period of fracture healing, but the degradation rate must match with the rate of bone healing. Cognition and ascertaining the laws of magnesium alloy degradation affected by different mechanical environments is the problem of magnesium alloys applied to clinical must be solved. The domestic and overseas researches on the degradation of magnesium alloy under mechanical circumstance are few. Gu [6] studied corrosion fatigue performance of AZ91D and WE43 magnesium alloy under cyclic loading. Study showed that the cyclic loading would accelerate the corrosion rate, and corrosion rate increased with the load. Hazibullah Waizy etc [7] studied mechanical properties of magnesium alloy affected by corrosive environment. Experiments used $\mathrm{MgCa} 0.8$ screw as the research object, which was fixed on the natural bone, using Hank's liquid corrosion environment. Pull-out force experiment was conducted at the conditions of non-corrosion and corrosion of after 24 , 48, 72 and 96 hours. Results showed that corrosion after a certain period of time, the mechanical properties decreased significantly. The research showed that [11] establishing ideal mechanical environment can promote fracture healing. As biomedical materials, magnesium alloys degradation rate must be controlled reasonably in vivo, so that it can maintain mechanical integrity of the bone tissue and give certain stress within a certain period of time, and degrading slowly in the bone tissue after recovery. This experiment is the qualitative experiment of the effect of mechanical factors on degradation of magnesium alloy, and experimental results showed that mechanical environment has impact on degradation of magnesium alloy. Current study of the biocompatibility of magnesium alloy has been improved, however, magnesium alloy bone internal fixation material applied into clinic and benefit to majority of patients, must be controlled in the degradation rate. In this process, degradation laws of magnesium alloys should be researched quantitatively. This experiment is fundamental research of magnesium alloy degradation affect by mechanical environment, which laid a foundation for the further study of biodegradable magnesium alloy.

5 Acknowledgements: national natural fund (11172208,11372221)

\section{Reference}

1. Fu Dongwei, Yan Yuhua.Biodegradable medical materials, The research progress of biological bone materials and clinical research, 2, 39(2005)

2. Strager M P,Pietak A M,Huadmai J,et a1.Magnesium and its alloys as orthopedic biomaterials.A reviewEJ],Biomaterials,27(2006),

3. Zhang Ye.Application of magnesium alloy in implantable medical devices,Industrial technology, 5(2012)

4. Jiang dongmei,Cao Zhanyi.Mg - zinc - Ca - Zr/Nd/Y biological magnesium alloy organization.,mechanical properties and corrosion behavior research, Jilin university, 6(2014)

5. Witter F:'Thistory of biodegradable magnesium implants-A eview',Actabiomater. 6(2010)

6. Gu XN, Zhou WR, Zheng YF, et al.Corrosion fatigue behaviors of two biomedical Mg alloys-AZ91D and WE43 In simulated body fluid,Acta Biomater,6(2010)

7. Hazibullah W, Andreas W.Biomechanical characterisation of a degradable magnesium-based (MgCa0.8) scre,J Mater Sci, Mater Med, (2012)

8. Song G, Atrens A, Stjohn D, etal.The electrochemical corrosion of pure magnesium in $\mathrm{NaCl}$. corros Sci, 5(1997)

9. Zhang Xiao, Liang Minjie , Liao Haihong, pei-kang ,Bai Peikang,Huang Sha:The research progress of biomedical magnesium alloy corrosion and protection, The hot working processes,4( 2014)

10. Wang Yongping, Yin Zifei,Jiang-zhuang, He Yao-hua.The application of magnesium alloy materials in the field of clinical medicine', Chinese journal of clinical physicians, 12(2011)

11. Xing Dan,Ma Xinlong etc.The research status of long bone fracture healing mechanics environment. Biomedical engineering and clinical, 11(2012) 